\title{
Entanglement Wedges from the Information Metric in Conformal Field Theories
}

\author{
Yuki Suzuki, ${ }^{1}$ Tadashi Takayanagi, ${ }^{2,3}$ and Koji Umemoto ${ }^{2}$ \\ ${ }^{1}$ Faculty of Science, Kyoto University, Kitashirakawa Oiwakecho, Sakyo-ku, Kyoto 606-8502, Japan \\ ${ }^{2}$ Center for Gravitational Physics, Yukawa Institute for Theoretical Physics, Kyoto University, \\ Kitashirakawa Oiwakecho, Sakyo-ku, Kyoto 606-8502, Japan \\ ${ }^{3}$ Kavli Institute for the Physics and Mathematics of the Universe (WPI), University of Tokyo, \\ Kashiwa, Chiba 277-8582, Japan
}

(Received 2 September 2019; published 26 November 2019)

\begin{abstract}
We present a new method of deriving the geometry of entanglement wedges in holography directly from conformal field theories (CFTs). We analyze an information metric called the Bures metric of reduced density matrices for locally excited states. This measures the distinguishability of states with different points excited. For a subsystem given by an interval, we precisely reproduce the expected entanglement wedge for two-dimensional holographic CFTs from the Bures metric, which turns out to be proportional to the anti-de Sitter metric on a time slice. On the other hand, for free scalar CFTs, we do not find any sharp structures like entanglement wedges. When a subsystem consists of two disconnected intervals, we manage to reproduce the expected entanglement wedge from holographic CFTs with the correct phase transitions, up to a very small error, from a quantity alternative to the Bures metric.
\end{abstract}

DOI: 10.1103/PhysRevLett.123.221601

Introduction.-An important and fundamental question in the AdS/CFT correspondence [1] is whether physical information included in a given region $A$ in conformal field theory (CFT) can correspond to that in a certain region $M_{A}$ in anti-de Sitter (AdS) gravity. More concretely, here we mean that the reduced density matrix $\rho_{A}$ of a CFT on a subregion $A$ is equivalent to a reduced density matrix $\rho_{M_{A}}^{\text {bulk }}$ of bulk gravity on a certain region $M_{A}$ in AdS. The answer to this question has been argued to be yes, where $M_{A}$ is given by the entanglement wedge [2-4], i.e., the region surrounded by the subsystem $A$ and the extremal surface $\Gamma_{A}$, whose area gives the holographic entanglement entropy $[5,6]$.

Normally this subregion duality is explained by combining several ideas: the gravity dual of a bulk field operator (i.e., Hamilton-Kabat-Lifschytz-Lowe map [7]), quantum corrections to holographic entanglement entropy $[8,9]$, and the conjectured connection between AdS/CFT and quantum error correcting codes [10,11]. However, since these highly employ the dual AdS geometry from the beginning, it is not clear how entanglement wedges emerge from CFT itself. The main aim of this Letter is to derive the geometry of an entanglement wedge purely from CFT computations. We will focus on

Published by the American Physical Society under the terms of the Creative Commons Attribution 4.0 International license. Further distribution of this work must maintain attribution to the author(s) and the published article's title, journal citation, and DOI. Funded by SCOAP ${ }^{3}$. two-dimensional (2D) CFTs for technical reasons. The AdS/CFT argues that a special class of CFTs, called holographic CFTs, can have classical gravity duals, described by general relativity. A holographic CFT is characterized by a large central charge $c$ and strong interactions, which lead to a large spectrum gap [12,13]. Therefore, the entanglement wedge geometry is available only when we consider holographic CFTs as we will see later.

Consider a state in a 2D CFT on a complex plane $R^{2}$, locally excited by a primary operator $O(w, \bar{w})$. We set $(w, \bar{w})=(x+i \tau, x-i \tau)$. By choosing a subsystem $A$ on the $x$ axis, we define the reduced density matrix on $A$, tracing out its complement $B$,

$$
\rho_{A}(w, \bar{w})=\operatorname{Tr}_{B}\left[O(w, \bar{w})|0\rangle\langle 0| O^{\dagger}(\bar{w}, w)\right],
$$

first introduced in [14] to study its entanglement entropy.

We assume that the conformal dimension $h$ of $O$ satisfies $1 \ll h \ll c$. Thus, we can neglect its backreaction in the gravity dual and can approximate the two-point function $\left\langle O\left(w_{1}, \bar{w}_{1}\right) O^{\dagger}\left(w_{2}, \bar{w}_{2}\right)\right\rangle$ by the geodesic length in the gravity dual between the two points $\left(w_{1}, \bar{w}_{1}\right)$ and $\left(w_{2}, \bar{w}_{2}\right)$ on the boundary $\eta \rightarrow 0$ of Poincaré $\mathrm{AdS}_{3}$,

$d s^{2}=\eta^{-2}\left(d \eta^{2}+d w d \bar{w}\right)=\eta^{-2}\left(d \eta^{2}+d x^{2}+d \tau^{2}\right)$.

Therefore, by projecting on the bulk time slice $\tau=0$, the state $\rho_{A}(w, \bar{w})$ is dual to a bulk excitation at a bulk point $P$ defined by the intersection between the time slice $\tau=0$ and the geodesic, as in Fig. 1. 


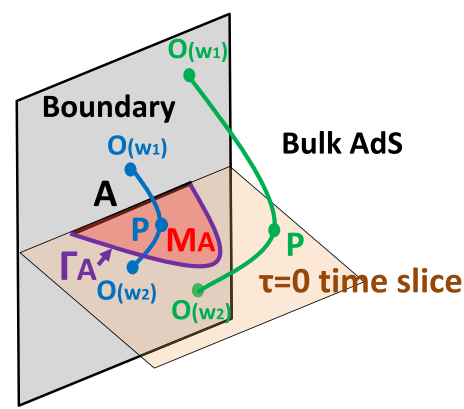

FIG. 1. A sketch of entanglement wedge $M_{A}$ (red colored region) for an interval $A$ in $\mathrm{AdS}_{3} / \mathrm{CFT}_{2}$ and holographic computations of two-point functions dual to geodesics.

Now we are interested in how we can distinguish the two states: $\rho_{A}(w, \bar{w})$ and $\rho_{A}\left(w^{\prime}, \bar{w}^{\prime}\right)$ when $w \neq w^{\prime}$, created by the same operators. On the time slice $\tau=0$, the location of dual bulk excitations is given by $(\eta, x)=(\tau, x)$ and $(\eta, x)=\left(\tau^{\prime}, x^{\prime}\right)$. The entanglement wedge reconstruction argues that we cannot distinguish the two excited bulk states when both excitations are outside of $M_{A}$, while we can distinguish them if at least one of them is inside of $M_{A}$.

A useful measure of distinguishability between two density matrices $\rho$ and $\rho^{\prime}$ is the Bures distance $D_{B}$, defined by (refer to, e.g., [15])

$$
D_{B}\left(\rho, \rho^{\prime}\right)^{2}=2\left(1-\operatorname{Tr}\left[\sqrt{\sqrt{\rho} \rho^{\prime} \sqrt{\rho}}\right]\right),
$$

When the density matrix is parametrized by continuous valuables $\lambda^{i}$, denoted by $\rho(\lambda)$, we can define the information metric

$$
D_{B}[\rho(\lambda+d \lambda), \rho(\lambda)]^{2} \simeq G_{i j} d \lambda^{i} d \lambda^{j} \equiv d D_{B}^{2},
$$

where $d \lambda_{i}$ are infinitesimally small. This metric $G_{i j}$ is called the Bures metric, which measures the distinguishability between nearby states.

The quantum version of the Cramér-Rao theorem [16] tells us that, when we estimate the values of $\lambda_{i}$, the errors of measurements are bounded as follows:

$$
\left\langle\left\langle\delta \lambda^{i} \delta \lambda^{j}\right\rangle\right\rangle \geq\left(G^{-1}\right)^{i j}
$$

As an exercise, consider the case where $A$ covers the total system, where $\rho_{A}(w, \bar{w})$ becomes a pure state $|\phi(w)\rangle\langle\phi(w)|$. The Bures distance $D_{B}$ is simplified as

$$
\begin{aligned}
D_{B}\left(|\phi\rangle,\left|\phi^{\prime}\right\rangle\right)^{2} & =2\left[1-\left|\left\langle\phi(w) \mid \phi\left(w^{\prime}\right)\right\rangle\right|\right], \\
\left|\left\langle\phi(w) \mid \phi\left(w^{\prime}\right)\right\rangle\right| & =|w-\bar{w}|^{2 h}\left|w^{\prime}-\bar{w}^{\prime}\right|^{2 h}\left|w-\bar{w}^{\prime}\right|^{-4 h} .
\end{aligned}
$$

This leads to the Bures metric

$$
d D_{B}^{2}=\frac{h}{\tau^{2}}\left(d \tau^{2}+d x^{2}\right) .
$$

This is proportional to the time slice metric in the gravity dual (2). This coincidence is very natural because the distinguishability should increase as the bulk points are geometrically separated, as noted in [17]. However, this result is universal for any 2D CFTs.

Also, we often calculate (introduced in [18])

$$
I\left(\rho, \rho^{\prime}\right)=\frac{\operatorname{Tr}\left[\rho \rho^{\prime}\right]}{\sqrt{\operatorname{Tr}\left[\rho^{2}\right] \operatorname{Tr}\left[\rho^{\prime 2}\right]}}
$$

instead of $D_{B}$ to estimate distinguishability. We always find $0 \leq I\left(\rho, \rho^{\prime}\right) \leq 1$. We have $I\left(\rho, \rho^{\prime}\right)=1$ if and only if $\rho=\rho^{\prime}$.

Single interval case. - We choose the subsystem $A$ to be an interval $0 \leq x \leq L$ at $\tau=0$. The surface $\Gamma_{A}$ in the bulk $\operatorname{AdS}$ is the semicircle $(x-L / 2)^{2}+\eta^{2}=L^{2} / 4$. Thus, if the entanglement reconstruction is correct, the information metric vanishes if the bulk point $P$ is outside of the entanglement wedge. This boundary of this wedge in the CFT is given by

$$
|w-L / 2|=L / 2 .
$$

In this example, this is the same as the causal wedge [19].

Let us start with the calculation of the quantity $I\left(\rho, \rho^{\prime}\right)$ defined by (8), for $\rho=\rho_{A}(w, \bar{w})$ and $\rho^{\prime}=\rho_{A}\left(w^{\prime}, \bar{w}^{\prime}\right)$. Since this calculation is essentially that of $\operatorname{Tr}\left[\rho \rho^{\prime}\right]$, we perform the conformal transformation $z^{2}=w /(w-L)$, which maps two flat space path integrals that produce $\rho(w, \bar{w})$ and $\rho\left(w^{\prime}, \bar{w}^{\prime}\right)$ into a single plane ( $z$ plane). Refer to [20,21] for similar calculations. The insertion points of the four primary operators on the $z$ plane are

$z_{1}=\sqrt{\frac{-x-i \tau}{L-x-i \tau}}(\equiv z), \quad z_{2}=\sqrt{\frac{-x+i \tau}{L-x+i \tau}}(\equiv \bar{z})$,

$z_{3}^{\prime}=-\sqrt{\frac{-x^{\prime}-i \tau^{\prime}}{L-x^{\prime}-i \tau^{\prime}}}\left(\equiv-z^{\prime}\right), \quad z_{4}^{\prime}=-\sqrt{\frac{-x^{\prime}+i \tau^{\prime}}{L-x^{\prime}+i \tau^{\prime}}}\left(\equiv-\bar{z}^{\prime}\right)$.

Refer to Fig. 2 for this conformal mapping. It is important to note that the boundaries of the wedges (9) on the $w$ planes are mapped into the diagonal lines $z= \pm i \bar{z}$.

The quantity $\operatorname{Tr}\left[\rho \rho^{\prime}\right]$ is expressed as a correlation function on the $z$ plane,

$$
\begin{aligned}
& \operatorname{Tr}\left[\rho \rho^{\prime}\right]=\left(\left|\frac{d z_{1}}{d w_{1}}\right|\left|\frac{d z_{2}}{d w_{2}}\right|\left|\frac{d z_{3}^{\prime}}{d w_{3}^{\prime}}\right|\left|\frac{d z_{4}^{\prime}}{d w_{4}^{\prime}}\right|\right)^{2 h} \frac{H\left(z_{1}, z_{2}, z_{3}^{\prime}, z_{4}^{\prime}\right) Z^{(2)}}{\left(Z^{(1)}\right)^{2}}, \\
& H\left(z_{1}, z_{2}, z_{3}^{\prime}, z_{4}^{\prime}\right) \\
& \equiv \frac{\left\langle O^{\dagger}\left(z_{1}, \bar{z}_{1}\right) O\left(z_{2}, \bar{z}_{2}\right) O^{\dagger}\left(z_{3}^{\prime}, \bar{z}_{3}^{\prime}\right) O\left(z_{4}^{\prime}, \bar{z}_{4}^{\prime}\right)\right\rangle}{\left\langle O^{\dagger}\left(w_{1}, \bar{w}_{1}\right) O\left(w_{2}, \bar{w}_{2}\right)\right\rangle\left\langle O^{\dagger}\left(w_{3}^{\prime}, \bar{w}_{3}^{\prime}\right) O\left(w_{4}^{\prime}, \bar{w}_{4}^{\prime}\right)\right\rangle},
\end{aligned}
$$

where we write the vacuum partition function on a $n$ sheeted complex plane by $Z^{(n)}$. Finally, we obtain 


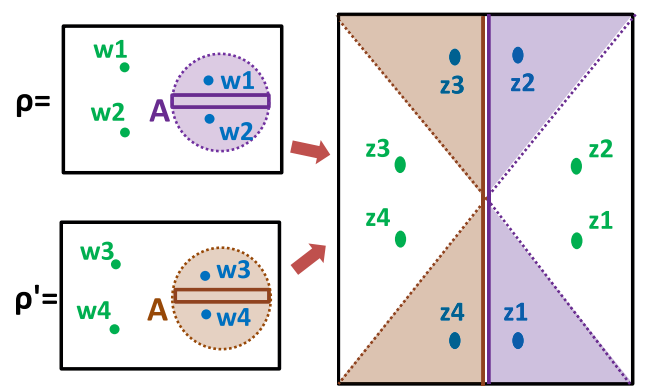

FIG. 2. A sketch of conformal transformation for the calculation of $\operatorname{Tr}\left[\rho \rho^{\prime}\right]$.

$$
\begin{aligned}
I\left(\rho, \rho^{\prime}\right) & =\frac{F\left(z_{1}, z_{2}, z_{3}^{\prime}, z_{4}^{\prime}\right)}{\sqrt{F\left(z_{1}, z_{2}, z_{3}, z_{4}\right) F\left(z_{1}^{\prime}, z_{2}^{\prime}, z_{3}^{\prime}, z_{4}^{\prime}\right)}}, \\
F\left(z_{1}, z_{2}, z_{3}^{\prime}, z_{4}^{\prime}\right) & \equiv\left\langle O^{\dagger}\left(z_{1}, \bar{z}_{1}\right) O\left(z_{2}, \bar{z}_{2}\right) O^{\dagger}\left(z_{3}^{\prime}, \bar{z}_{3}^{\prime}\right) O\left(z_{4}^{\prime}, \bar{z}_{4}^{\prime}\right)\right\rangle .
\end{aligned}
$$

In holographic CFTs, we can approximate the correlation functions by regarding the operators as generalized free fields [22] so that we simply take the Wick contractions of two-point functions (we set $z=z_{1}$ and $z^{\prime}=-z_{3}$ ),

$$
F\left(z_{1}, z_{2}, z_{3}^{\prime}, z_{4}^{\prime}\right) \simeq|z-\bar{z}|^{-4 h}\left|z^{\prime}-\bar{z}^{\prime}\right|^{-4 h}+\left|z+\bar{z}^{\prime}\right|^{-8 h} .
$$

The value of $I\left(\rho, \rho^{\prime}\right)$ as a function of $w^{\prime}=x^{\prime}+i \tau^{\prime}$ is plotted in the left two graphs in Fig. 3. The upper left graph is the case where $w$ is inside the wedge (9) and we have $I=1$ if and only if $w=w^{\prime}$, while $0<I<1$ if and only if $w \neq w^{\prime}$, as expected. This shows that we can correctly distinguish the states. On the other hand, if $w$ is outside the wedge (see the lower left graph), we find $I \simeq 1$ (i.e., indistinguishable)

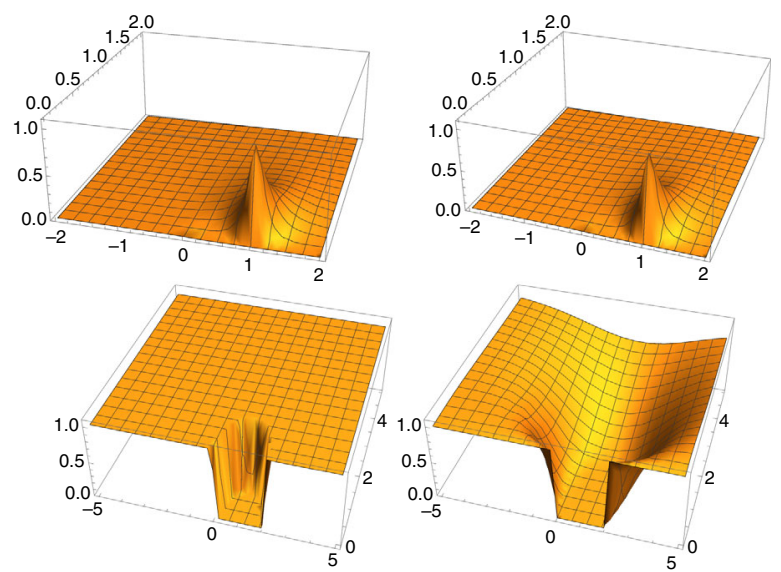

FIG. 3. The profiles of $I\left(\rho, \rho^{\prime}\right)$ as a function of $x^{\prime}$ (horizontal axis) and $\tau^{\prime}$ (depth axis) for the choice $A=[0,2]$ (i.e., $L=2$ ). The two left ones are for a 2D holographic CFT, while the right ones for a 2D free scalar CFT. In the upper two graphs, we chose $h=1 / 2$ and $(x, \tau)=(1,0.1)$ and in the lower two, we chose $h=10$ and $(x, \tau)=(-1,0.1)$. if $w^{\prime}$ is also outside, while we have $I \simeq 0$ if $w^{\prime}$ is inside. We can see that the border is precisely the CFT counterpart of the entanglement wedge (9). This border gets very sharp when $h \gg 1$ as we are assuming for geodesic approximation. These behaviors perfectly agree with bulk state distinguishability in AdS/CFT.

When we calculate the information metric, we assume $w \simeq w^{\prime}$ (or equally $z \simeq z^{\prime}$ ). In this case, the first term in (11) dominates when $|z-\bar{z}| \leq|z+\bar{z}|$ and this condition precisely matches that for the outside wedge condition. Indeed, if we only keep this first term, we immediately find $I\left(\rho, \rho^{\prime}\right)=1$. On the other hand, when it is inside, the second term is dominant and the result is identical to the case where $A$ is the total space [i.e., $\rho_{A}=|\phi(w)\rangle\langle\phi(w)|$ is pure].

As a comparison, in the right two graphs in Fig. 3, we plotted $I\left(\rho, \rho^{\prime}\right)$ for a $2 \mathrm{D}$ free massless scalar CFT (the scalar field is denoted by $\varphi$ ), where we chose the primary operator to be $O(w, \bar{w})=e^{i \alpha \rho(w, \bar{w})}$, which has the dimension $h=\alpha^{2} / 2$. Clearly, in this free CFT, we cannot find any sharp structure of an entanglement wedge as opposed to holographic CFTs, though they have qualitative similarities.

We can evaluate the Bures metric from

$$
A_{n, m}=\operatorname{Tr}\left[\left(\rho^{m} \rho^{\prime} \rho^{m}\right)^{n}\right]
$$

via the analytical continuation $n=m=1 / 2$. We apply the conformal transformation [we set $k=(2 m+1) n$ ] $z^{k}=$ $w /(w-L)$, so that the path integrals for $2 m n \rho$ 's and $n \rho^{\prime}$ 's are mapped into that on a single plane. This leads to

$A_{n, m}=\frac{\left\langle O^{\dagger}\left(w_{1}\right) O\left(w_{2}\right) \cdots O^{\dagger}\left(w_{2 k-1}\right) O\left(w_{2 k}\right)\right\rangle Z^{(k)}}{\prod_{i=1}^{k}\left\langle O^{\dagger}\left(w_{2 i-1}\right) O\left(w_{2 i}\right)\right\rangle\left(Z^{(1)}\right)^{k}}$.

Refer to $[23,24]$ for analogous computations of relative entropy. Then we find

$$
\begin{aligned}
A_{n, m}= & \prod_{i=1}^{2 k}\left|k^{-1}\left(z_{i}\right)^{1-k}\right|^{2 h} \prod_{j=1}^{k}\left|\left(z_{2 j-1}\right)^{k}-\left(z_{2 j}\right)^{k}\right|^{4 h} \\
& \times\left\langle O^{\dagger}\left(z_{1}\right) O\left(z_{2}\right) \cdots O^{\dagger}\left(z_{2 k-1}\right) O\left(z_{2 k}\right)\right\rangle \frac{Z^{(k)}}{\left(Z^{(1)}\right)^{k}} .
\end{aligned}
$$

Note that we have

$$
\begin{aligned}
z_{1} & =\left(\frac{-x-i \tau}{L-x-i \tau}\right)^{1 / k}, \quad z_{2}\left(=\bar{z}_{1}\right)=\left(\frac{-x+i \tau}{L-x+i \tau}\right)^{1 / k}, \\
z_{2 s+1} & =e^{(2 \pi i / k) s} z_{1}, \quad z_{2 s+2}=e^{(2 \pi i / k) s} z_{2}, \quad(s=1,2, \ldots, k-1) .
\end{aligned}
$$

Let us evaluate $A_{n, m}$ in holographic CFTs, using the generalized free field approximation. We take $w \simeq w^{\prime}$ to calculate the Bures metric. When $w$ and $w^{\prime}$ are outside of the entanglement wedge (9), or equally $\left|z_{2 j-1}-z_{2 j}\right|<$ $\left|z_{2 j-2}-z_{2 j-1}\right|$, the $2 k$ point function is approximated as 


$$
\begin{aligned}
& \left\langle O^{\dagger}\left(z_{1}\right) O\left(z_{2}\right) \cdots O^{\dagger}\left(z_{2 k-1}\right) O\left(z_{2 k}\right)\right\rangle \\
& \quad \simeq \prod_{j=1}^{k}\left\langle O^{\dagger}\left(z_{2 j-1}\right) O\left(z_{2 j}\right)\right\rangle \simeq \prod_{j=1}^{k}\left|z_{2 j-1}-z_{2 j}\right|^{-4 h},
\end{aligned}
$$

and this leads to the trivial result $A_{n, m}=1$, leading to the vanishing Bures metric $d D_{B}^{2}=0$. This agrees with the AdS/CFT expectation that $\rho_{A}$ cannot distinguish two different bulk excitations outside of entanglement wedge.

On the other hand, when $w$ and $w^{\prime}$ are inside of the entanglement wedge (9), or equally $\left|z_{2 j-1}-z_{2 j}\right|>$ $\left|z_{2 j-2}-z_{2 j-1}\right|$, we can approximate as

$$
\begin{aligned}
& \left\langle O^{\dagger}\left(z_{1}\right) O\left(z_{2}\right) \cdots O^{\dagger}\left(z_{2 k-1}\right) O\left(z_{2 k}\right)\right\rangle \\
& \quad \simeq \prod_{j=1}^{k}\left\langle O^{\dagger}\left(z_{2 j-2}\right) O\left(z_{2 j-1}\right)\right\rangle \simeq \prod_{j=1}^{k}\left|z_{2 j-2}-z_{2 j-1}\right|^{-4 h} .
\end{aligned}
$$

In the limit $n \rightarrow 1 / 2$ and $m \rightarrow 1 / 2$, this leads to

$$
\begin{aligned}
A_{1 / 2,1 / 2} & =|w-\bar{w}|^{2 h}\left|w^{\prime}-\bar{w}^{\prime}\right|^{2 h}\left|w^{\prime}-\bar{w}\right|^{-4 h}, \\
d D_{B}^{2} & =\frac{h}{\tau^{2}}\left(d x^{2}+d \tau^{2}\right) .
\end{aligned}
$$

This Bures metric for $\rho_{A}(w, \bar{w})$ coincides with that for the pure state (7) and reproduces the bulk AdS metric on the time slice $\tau=0$.

Similarly, in a 2D holographic CFT with a circle compactification $x \sim x+2 \pi$, we obtain the Bures metric

$$
d D_{B}^{2}=\frac{h}{\sinh ^{2} \tau}\left(d \tau^{2}+d x^{2}\right),
$$

if $w$ is inside the wedge. In a $2 \mathrm{D}$ holographic CFT at finite temperature $T$, we obtain

$$
d D_{B}^{2}=h \frac{(2 \pi T)^{2}}{\sin ^{2}(2 \pi T \tau)}\left(d \tau^{2}+d x^{2}\right)
$$

These metrics agree with those on the time slice $\tau=0$ of global $\mathrm{AdS}_{3}$ and Banados-Teitelboim-Zanelli (BTZ) black hole, by projecting boundary points $(x, \tau)$ into the time slice along geodesics.

It is instructive to calculate the Bures metric in the 2D massless free scalar CFT for the primary $O=e^{i \alpha \varphi}$. For $\alpha=1$, we find the following analytical result:

$$
\begin{aligned}
A_{1 / 2,1 / 2}= & \frac{\left(\sqrt{z}+\sqrt{z^{\prime}}\right)\left(\sqrt{\bar{z}}+\sqrt{\overline{z^{\prime}}}\right)(\sqrt{z}+\sqrt{\bar{z}})\left(\sqrt{z^{\prime}}+\sqrt{\bar{z}^{\prime}}\right)}{4 \sqrt{|z|\left|z^{\prime}\right|}\left(\sqrt{z}+\sqrt{\bar{z}^{\prime}}\right)\left(\sqrt{\bar{z}}+\sqrt{z^{\prime}}\right)} \\
d D_{B}^{2}= & -\frac{L^{2}(d w)^{2}}{16 w^{2}(L-w)^{2}}-\frac{L^{2}(d \bar{w})^{2}}{16 \bar{w}^{2}(L-\bar{w})^{2}} \\
& +\frac{L^{2}(d w)(d \bar{w})}{2|w||w-L|(\sqrt{\bar{w}(w-L)}+\sqrt{w(\bar{w}-L)})^{2}}
\end{aligned}
$$

Note that we cannot find any sharp structure of an entanglement wedge as opposed to the holographic CFT.

Double interval case.-Next we take the subsystem $A$ to be a union of two disconnected intervals $A_{1}$ and $A_{2}$, which are parametrized as $A_{1}=[0, s]$ and $A_{2}=[l+s, l+2 s]$, without losing generality. We conformally map the $w$ plane with two slits along $A_{1}$ and $A_{2}$ into a $z$ cylinder via (see, e.g., [25])

$$
z=f(w)=\frac{J\left(\kappa^{2}\right)}{2}-\frac{J\left(\kappa^{2}\right)}{2 K\left(\kappa^{2}\right)} \operatorname{sn}^{-1}\left(\tilde{w}, \kappa^{2}\right),
$$

where we introduced

$$
\begin{aligned}
\tilde{w} & =\frac{2}{l}\left(w-s-\frac{l}{2}\right), \quad J\left(\kappa^{2}\right)=2 \pi \frac{K\left(\kappa^{2}\right)}{K\left(1-\kappa^{2}\right)}, \\
K\left(\kappa^{2}\right) & =\int_{0}^{1} \frac{d x}{\sqrt{\left(1-x^{2}\right)\left(1-\kappa^{2} x^{2}\right)}}, \quad \kappa=\frac{l}{l+2 s} .
\end{aligned}
$$

The function $\operatorname{sn}^{-1}\left(\tilde{w}, \kappa^{2}\right)$ is the Jacobi elliptic function.

We can calculate $I\left(\rho, \rho^{\prime}\right)$ using the map (20) both for $\rho_{A}(w, \bar{w})$ and $\rho_{A}\left(w^{\prime}, \bar{w}^{\prime}\right)$ and the formula (10). The two $w$ planes are mapped into a torus, described by the $z$ plane with the identification $\operatorname{Re}[z] \sim \operatorname{Re}[z]+2 J$ and $\operatorname{Im}[z] \sim$ $\operatorname{Im}[z]+2 \pi$, as depicted in Fig. 4 .

In holographic CFTs, we need to distinguish two phases depending on the moduli of the torus: (i) the connected

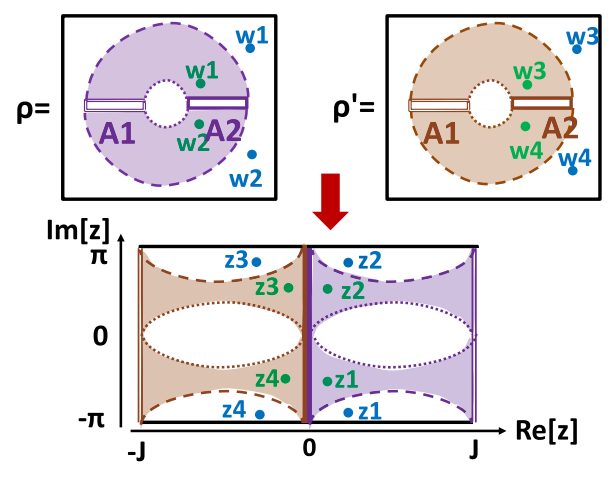

FIG. 4. A sketch of conformal transformation for $\operatorname{Tr}\left[\rho_{A} \rho_{A}^{\prime}\right]$ in the double interval case. We assumed the phase (i), where the entanglement wedge is connected, as depicted by the colored region. The lower picture describes the geometry after the transformation and is given by a torus by identifying the edges. Blue (or green) points are outside (or inside) of $M_{A}$. 
phase, $J<\pi$ or equally $\kappa<3-2 \sqrt{2}$, and (ii) the disconnected phase, $J>\pi$ or equally $\kappa>3-2 \sqrt{2}$. We can confirm phase (i) [or (ii)] coincides with the case in the gravity dual where the entanglement wedge gets connected (or disconnected), and the circle $\operatorname{Re}[z]$ (or $\operatorname{Im}[z]$ ) shrinks to zero size in the bulk, respectively. This is the standard Hawking-Page transition [26] and agrees with the large $c$ CFT analysis [12]. The holographic two-point functions on the torus in each phase behave like

$$
\begin{aligned}
\left\langle O^{\dagger}(z, \bar{z}) O\left(z^{\prime}, \bar{z}^{\prime}\right)\right\rangle_{(i)} & \simeq \max _{n_{1} \in Z}\left|\sin \left(\frac{\pi\left(z+2 \pi i n_{1}-z^{\prime}\right)}{2 J}\right)\right|^{-4 h}, \\
\left\langle O^{\dagger}(z, \bar{z}) O\left(z^{\prime}, \bar{z}^{\prime}\right)\right\rangle_{(i i)} & \simeq \max _{n_{2} \in Z}\left|\sinh \left(\frac{\left(z+2 J n_{2}-z^{\prime}\right)}{2}\right)\right|^{-4 h} .
\end{aligned}
$$

Let us estimate four-point functions $F$ in (10) by the generalized free field prescription, where we again assume $w \simeq w^{\prime}$. There are two contributions: the trivial Wick contraction and the nontrivial one as in (11). The trivial one leads to $I\left(\rho, \rho^{\prime}\right)=1$, which tells us that we cannot distinguish the two states. Therefore, we again find that the entanglement wedge corresponds to the region where nontrivial contractions become dominant.

The nontrivial Wick contraction is dominant when

$\min _{n_{1} \in Z}\left|\sin \left(\frac{\pi}{2 J}\left(z_{2}-z_{1}-2 n_{1} \pi i\right)\right)\right| \geq\left|\sin \left(\frac{\pi}{2 J}\left(z_{3}-z_{2}\right)\right)\right|$

in the connected case and when

$$
\left|\sinh \left(\frac{z_{2}-z_{1}}{2}\right)\right| \geq \min _{n_{2} \in Z}\left|\sinh \left(\frac{z_{2}-z_{3}-2 n_{2} J}{2}\right)\right|
$$

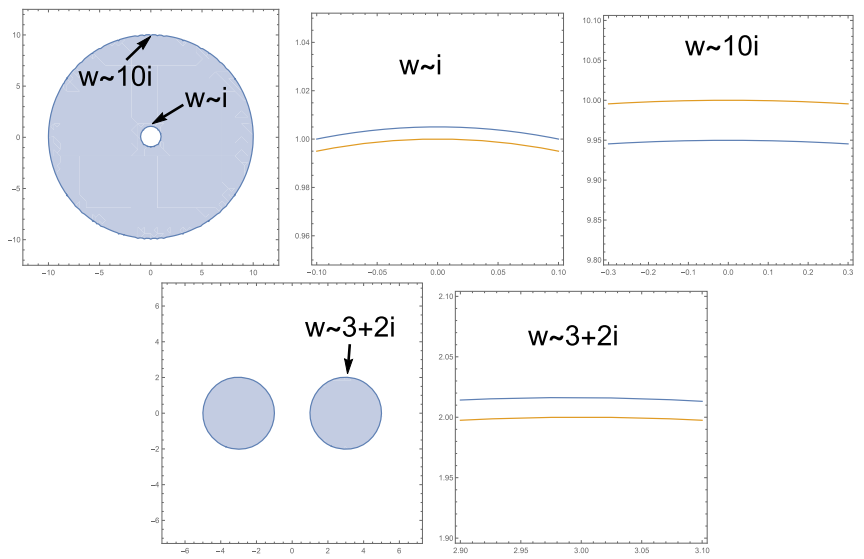

FIG. 5. The plots of the locations of the operator insertion on the $\tilde{w}$ plane where the nontrivial Wick contraction is favored (blue colored regions in the left pictures). The upper pictures are for $\kappa=0.1$ where $M_{A}$ is connected, while the lower ones are for $\kappa=$ 0.2 where $M_{A}$ is disconnected. In the upper middle and right pictures, blue curves are the borders between the nontrivial and trivial contraction, while orange curves describe the borders of the entanglement wedge. The same is true for the lower right picture. in the disconnected case. We plotted these regions in terms of the coordinate $\tilde{w}$ of (20) in Fig. 5.

In both cases, the regions are very close to correct entanglement wedges, respecting the expected connected or disconnected geometry (note our holographic relation in Fig. 1). However, there is a very small deviation, which is always within a few percent. This deviation arises because the correct distinguishability should be measured by the Bures metric. Our analysis using $I\left(\rho, \rho^{\prime}\right)$ only gives an approximation, much like the Renyi entropy compared with the von Neumann entropy.

The calculation of the genuine Bures distance $D_{B}\left(\rho, \rho^{\prime}\right)$ is very complicated, as the trace $\operatorname{Tr}\left[\left(\rho^{m} \rho^{\prime} \rho^{m}\right)^{n}\right]$ corresponds to a partition function on a genus $n(2 m+1)-1$ Riemann surface. However, in the final $n=m \rightarrow 1 / 2$ limit (genus zero limit), it might not be surprising to obtain the expected metric (7).

Conclusions.-In this Letter, we present a general mechanism to show how entanglement wedges emerge from holographic CFTs. One important future problem is to repeat the same procedure by using the genuine localized operator in the bulk [7] (see also [27,28] or the state [17]). Another interesting direction will be to extend this construction to the higher-dimensional AdS/CFT. Moreover, it may be useful to consider other distance measures such as trace distances [29]. It would also be intriguing to explore the relationship between our approach and the path-integral optimization [30]. It may also be fruitful to consider connections between our results and the recent proposals for entanglement wedge cross sections [31-36]. Additionally, it would be interesting to study $1 / N$ corrections in our setup, which might be related to quantum extremal surfaces [37]. We would like to revisit these problems soon [38].

We thank Pawel Caputa, Veronika Hubeny, Henry Maxfield, Mukund Rangamani, Hiroyasu Tajima, and Kotaro Tamaoka for useful conversations. T. T. is supported by the Simons Foundation through the "It from Qubit" collaboration. T. T. is supported by JSPS Grantin-Aid for Scientific Research (A) No. 16H02182 and by JSPS Grant-in-Aid for Challenging Research (Exploratory) 18K18766. T. T. is also supported by World Premier International Research Center Initiative (WPI Initiative) from the Japan Ministry of Education, Culture, Sports, Science, and Technology (MEXT). K. U. is supported by Grant-in-Aid for JSPS Fellows No. 18J22888. We are grateful to the long-term workshop "Quantum Information and String Theory" (YITP-T-1903) held at Yukawa Institute for Theoretical Physics, Kyoto University, and participants for useful discussions. T. T. is very thankful for the workshop "Quantum Information in Quantum Gravity V," held at UC Davis, where this work was presented. 
[1] J. M. Maldacena, The large-N limit of superconformal field theories and supergravity, Adv. Theor. Math. Phys. 2, 231 (1998); The large-N limit of superconformal field theories and supergravity, Int. J. Theor. Phys. 38, 1113 (1999).

[2] B. Czech, J. L. Karczmarek, F. Nogueira, and M. Van Raamsdonk, The gravity dual of a density matrix, Classical Quantum Gravity 29, 155009 (2012).

[3] A. C. Wall, Maximin surfaces, and the strong subadditivity of the covariant holographic entanglement entropy, Classical Quantum Gravity 31, 225007 (2014).

[4] M. Headrick, V. E. Hubeny, A. Lawrence, and M. Rangamani, Causality and holographic entanglement entropy, J. High Energy Phys. 12 (2014) 162.

[5] S. Ryu and T. Takayanagi, Holographic Derivation of Entanglement Entropy from AdS/CFT, Phys. Rev. Lett. 96, 181602 (2006); Aspects of holographic entanglement entropy, J. High Energy Phys. 08 (2006) 045.

[6] V.E. Hubeny, M. Rangamani, and T. Takayanagi, A Covariant holographic entanglement entropy proposal, J. High Energy Phys. 07 (2007) 062.

[7] A. Hamilton, D. N. Kabat, G. Lifschytz, and D. A. Lowe, Holographic representation of local bulk operators, Phys. Rev. D 74, 066009 (2006).

[8] T. Faulkner, A. Lewkowycz, and J. Maldacena, Quantum corrections to holographic entanglement entropy, J. High Energy Phys. 11 (2013) 074.

[9] D. L. Jafferis, A. Lewkowycz, J. Maldacena, and S. J. Suh, Relative entropy equals bulk relative entropy, J. High Energy Phys. 06 (2016) 004.

[10] A. Almheiri, X. Dong, and D. Harlow, Bulk locality and quantum error correction in AdS/CFT, J. High Energy Phys. 04 (2015) 163; D. Harlow, The Ryu-Takayanagi formula from quantum error correction, Commun. Math. Phys. 354, 865 (2017).

[11] X. Dong, D. Harlow, and A. C. Wall, Reconstruction of Bulk Operators within the Entanglement Wedge in Gauge-Gravity Duality, Phys. Rev. Lett. 117, 021601 (2016).

[12] M. Headrick, Entanglement Renyi entropies in holographic theories, Phys. Rev. D 82, 126010 (2010).

[13] T. Hartman, C. A. Keller, and B. Stoica, Universal spectrum of $2 \mathrm{~d}$ conformal field theory in the large c limit, J. High Energy Phys. 09 (2014) 118.

[14] M. Nozaki, T. Numasawa, and T. Takayanagi, Quantum Entanglement of Local Operators in Conformal Field Theories, Phys. Rev. Lett. 112, 111602 (2014).

[15] M. Hayashi, Quantum Information Theory, Graduate Texts in Physics (Springer, New York, 2017).

[16] C. W. Helstrom, Minimum mean-square error estimation in quantum statistics, Phys. Lett. 25A, 101 (1967).

[17] M. Miyaji, T. Numasawa, N. Shiba, T. Takayanagi, and K. Watanabe, Continuous Multiscale Entanglement Renormalization Ansatz as Holographic Surface-State Correspondence, Phys. Rev. Lett. 115, 171602 (2015).

[18] J. Cardy, Thermalization and Revivals after a Quantum Quench in Conformal Field Theory, Phys. Rev. Lett. 112, 220401 (2014).

[19] V.E. Hubeny and M. Rangamani, Causal holographic information, J. High Energy Phys. 06 (2012) 114.
[20] M. Nozaki, T. Numasawa, and T. Takayanagi, Quantum Entanglement of Local Operators in Conformal Field Theories, Phys. Rev. Lett. 112, 111602 (2014); M. Nozaki, Notes on quantum entanglement of local operators, J. High Energy Phys. 10 (2014) 147.

[21] S. He, T. Numasawa, T. Takayanagi, and K. Watanabe, Quantum dimension as entanglement entropy in twodimensional conformal field theories, Phys. Rev. D 90, 041701(R) (2014).

[22] S. El-Showk and K. Papadodimas, Emergent spacetime and holographic CFTs, J. High Energy Phys. 10 (2012) 106.

[23] N. Lashkari, Relative Entropies in Conformal Field Theory, Phys. Rev. Lett. 113, 051602 (2014); Modular Hamiltonian for Excited States in Conformal Field Theory, Phys. Rev. Lett. 117, 041601 (2016).

[24] G. Srosi and T. Ugajin, Relative entropy of excited states in two dimensional conformal field theories, J. High Energy Phys. 07 (2016) 114.

[25] M. A. Rajabpour, Post measurement bipartite entanglement entropy in conformal field theories, Phys. Rev. B 92, 075108 (2015); Fate of the area-law after partial measurement in quantum field theories, arXiv:1503.07771; Entanglement entropy after partial projective measurement in $1+1$ dimensional conformal field theories: Exact results, J. Stat. Mech. (2016) 063109.

[26] S. W. Hawking and D. N. Page, Thermodynamics of black holes in anti-de Sitter Space, Commun. Math. Phys. 87, 577 (1983).

[27] D. Kabat and G. Lifschytz, Local bulk physics from intersecting modular Hamiltonians, J. High Energy Phys. 06 (2017) 120.

[28] S. R. Roy and D. Sarkar, Bulk metric reconstruction from boundary entanglement, Phys. Rev. D 98, 066017 (2018).

[29] J. Zhang, P. Ruggiero, and P. Calabrese, Subsystem Trace Distance in Quantum Field Theory, Phys. Rev. Lett. 122, 141602 (2019); Subsystem trace distance in low-lying states of conformal field theories, J. High Energy Phys. 10 (2019) 181.

[30] P. Caputa, N. Kundu, M. Miyaji, T. Takayanagi, and K. Watanabe, Anti-de Sitter Space from Optimization of Path Integrals in Conformal Field Theories, Phys. Rev. Lett. 119, 071602 (2017); Liouville action as path-integral complexity: From continuous tensor networks to AdS/CFT, J. High Energy Phys. 11 (2017) 097.

[31] K. Umemoto and T. Takayanagi, Entanglement of purification through holographic duality, Nat. Phys. 14, 573 (2018).

[32] P. Nguyen, T. Devakul, M. G. Halbasch, M. P. Zaletel, and B. Swingle, Entanglement of purification: From spin chains to holography, J. High Energy Phys. 01 (2018) 098.

[33] J. Kudler-Flam and S. Ryu, Entanglement negativity and minimal entanglement wedge cross sections in holographic theories, Phys. Rev. D 99, 106014 (2019).

[34] P. Caputa, M. Miyaji, T. Takayanagi, and K. Umemoto, Holographic Entanglement of Purification from Conformal Field Theories, Phys. Rev. Lett. 122, 111601 (2019). 
[35] K. Tamaoka, Entanglement Wedge Cross Section from the Dual Density Matrix, Phys. Rev. Lett. 122, 141601 (2019).

[36] S. Dutta and T. Faulkner, A canonical purification for the entanglement wedge cross-section, arXiv:1905.00577.
[37] N. Engelhardt and A. C. Wall, Quantum extremal surfaces: Holographic entanglement entropy beyond the classical regime, J. High Energy Phys. 01 (2015) 073.

[38] Y. Suzuki, T. Takayanagi, and K. Umemoto (to be published). 